\title{
Risk of Uterine Rupture and Placenta Accreta With Prior Uterine Surgery Outside of the Lower Segment
}

\author{
Cynthia Gyamfi-Bannerman, MD, Sharon Gilbert, MS, Mark B. Landon, MD, Catherine Y. \\ Spong, MD, Dwight J. Rouse, MD, Michael W. Varner, MD, Steve N. Caritis, MD, Paul J. \\ Meis, MD, Ronald J. Wapner, MD, Yoram Sorokin, MD, Marshall Carpenter, MD, Alan M. \\ Peaceman, MD, Mary J. O'Sullivan, MD, Baha M. Sibai, MD, John M. Thorp, MD, Susan M. \\ Ramin, MD, Brian M. Mercer, MD, and Eunice Kennedy Shriver National Institute of Child \\ Health and Human Development (NICHD) Maternal-Fetal Medicine Units (MFMU) Network \\ Departments of Obstetrics and Gynecology at Columbia University, New York, NY; The Ohio \\ State University, Columbus, $\mathrm{OH}$; University of Alabama at Birmingham, Birmingham, AL; \\ University of Utah, Salt Lake City, UT; University of Pittsburgh, Pittsburgh, PA; Wake Forest \\ University Health Sciences, Winston-Salem, NC; Thomas Jefferson University, Philadelphia, PA; \\ Wayne State University, Detroit, MI; Brown University, Providence, RI; Northwestern University, \\ Chicago, IL; University of Miami, Miami, FL; University of Tennessee, Memphis, TN; University of \\ North Carolina, Chapel Hill, NC; University of Texas Health Science Center at Houston, Houston, \\ TX; Case Western Reserve University-MetroHealth Medical Center, Cleveland, $\mathrm{OH}$; and The \\ George Washington University Biostatistics Center, Washington, DC; and the Eunice Kennedy \\ Shriver National Institute of Child Health and Human Development, Bethesda, MD
}

\begin{abstract}
Objective-Women with a prior myomectomy or prior classical cesarean delivery are often delivered early by cesarean due to concern for uterine rupture. Although theoretically at increased risk for placenta accreta, this risk has not been well quantified. Our objective was to estimate and compare the risks of uterine rupture and placenta accreta in women with prior uterine surgery.
\end{abstract}

\begin{abstract}
Methods-Women with prior myomectomy or prior classical cesarean delivery were compared to women with a prior low transverse cesarean to estimate rates of both uterine rupture and placenta accreta.
\end{abstract}

\begin{abstract}
Results-One hundred seventy-six women with a prior myomectomy, 455 with a prior classical cesarean delivery, and 13,273 women with a prior low transverse cesarean were evaluated. Mean gestational age at delivery differed by group $(\mathrm{p}<0.001)$, prior myomectomy $(37.3$ weeks), prior classical cesarean delivery ( 35.8 weeks), and low transverse cesarean (38.6 weeks). The frequency of uterine rupture in the prior myomectomy group was $0 \%$ (95\% CI 0-1.98\%). The frequency of uterine rupture in the low transverse cesarean group $(0.41 \%)$ was not statistically different from
\end{abstract}

(C) Copyright 2012 American College of Obstetricians and Gynecologists.

Corresponding Author: Cynthia Gyamfi-Bannerman, MD, Associate Clinical Professor, Columbia University Medical Center, Department of Obstetrics and Gynecology, Division of Maternal-Fetal Medicine, 622 West $168^{\text {th }}$ Street, PH-16, New York, NY 10032.cg2231@columbia.edu..

Presented at the Society for Gynecologic Investigation, March 24-27, 2010, Orlando, Florida.

*For a list of other members of the NICHD MFMU, see the Appendix online at http://links.lww.com/xxx.

Financial Disclosure: The authors did not report any potential conflicts of interest.

This is a PDF file of an unedited manuscript that has been accepted for publication. As a service to our customers we are providing this early version of the manuscript. The manuscript will undergo copyediting, typesetting, and review of the resulting proof before it is published in its final citable form. Please note that during the production process errors may be discovered which could affect the content, and all legal disclaimers that apply to the journal pertain. 
the risk in the prior myomectomy group ( $\mathrm{p}>0.99)$ or in the prior classical cesarean delivery group $(0.88 \%, \mathrm{p}=0.13)$. Placenta accreta occurred in $0 \%$ (95\% CI 0-1.98\%) of prior myomectomy compared with $0.19 \%$ in the low transverse cesarean group ( $p>0.99)$ and $0.88 \%$ in the prior classical cesarean delivery group ( $\mathrm{p}=0.01$ relative to low transverse cesarean). The adjusted OR for the prior classical cesarean delivery group (relative to low transverse cesarean) was 3.23 (1.11-9.39) for uterine rupture and 2.09 (0.69-6.33) for accreta. The frequency of accreta for those with previa was $11.1 \%$ for the prior classical cesarean delivery and $13.6 \%$ for low transverse cesarean groups ( $\mathrm{p}>0.99=1.0)$.

Conclusion-A prior myomectomy is not associated with higher risks of either uterine rupture or placenta accreta. The absolute risks of uterine rupture and accreta after prior myomectomy are low.

\section{INTRODUCTION}

The theoretical risk of uterine rupture before to the onset of labor in women with a prior classical cesarean delivery has led some practitioners to move the gestational age at delivery for these women into the late preterm period ( $340 / 7$ to 36 6/7 wks). ${ }^{1,2}$ Delivery of women with prior myomectomies has followed this trend because it is thought that the scar from a myomectomy is functionally equivalent to the scar from a classical cesarean. ${ }^{1,2}$ In practice there is a paucity of data on uterine rupture risk in women with a prior myomectomy. ${ }^{3-5}$ Similarly, there are very little data available about the rates of placenta accreta in women with prior uterine scars other than low segment transverse. Thus, our primary aim was to describe the risks of uterine rupture in women with a prior myomectomy, prior classical and prior lower segment cesarean, while our secondary aim was to compare risks of placenta accreta in these populations.

\section{METHODS}

This is a secondary analysis of the Cesarean Registry, a multicenter observational study of women undergoing both primary and repeat cesarean delivery as well as those attempting trial of labor after prior cesarean delivery. The study was conducted by the Eunice Kennedy Shriver National Institute of Child Health and Human Development (NICHD) MaternalFetal Medicine Units (MFMU) Network from 1999-2000 at 19 US academic centers. Full details of the methods and study design have been previously described. ${ }^{6}$ This secondary analysis was approved by the IRB at Columbia University Medical Center.

In the parent study, women delivered either vaginally or by cesarean. Uterine rupture was defined as a through and through disruption or tear of the uterine muscle and serosa; fetal contents may or may not have extruded into the abdomen. Placenta accreta was defined as a placenta that was adherent to the uterine wall without easy separation. In cases where a pathology report was available, it took precedence over the clinical findings. The definition of placenta previa was based on the presence of a clinician diagnosis of previa with or without bleeding after taking into consideration the clinical presentation and imaging results.

For this specific analysis, women with a singleton gestation and a prior myomectomy (PMMX), as reported by the patient and documented in the chart, or one prior cesarean delivery were included. Our sample size was fixed by available patients meeting inclusion criteria from the parent study. We excluded patients with both a prior myomectomy and a prior cesarean delivery. Additionally, women with a prior T or J uterine incision were excluded. The women with a prior cesarean delivery were divided into 2 groups by type of scar: prior classical cesarean delivery (PC) and prior low segment transverse cesarean (LTC). These women were compared to the P-MMX group to evaluate rates of both uterine 
rupture and placenta accreta. Type of prior scar was abstracted from the medical record supplemented by direct review of the operative reports when available.

We chose women with one prior low transverse cesarean as the reference group for the primary outcome. We compared uterine rupture in this group with women in the P-MMX and PC groups. Similarly, the LTC group was used as a reference for the secondary outcome, placenta accreta. Because it is known that placenta previa modifies the risk of placenta accreta, our final analysis evaluated rates of accreta in the three groups when a previa was present.

For statistical analyses, categorical variables were compared using the chi-square test or Fisher's exact test for dichotomous variables and the Kruskall-Wallace test for continuous variables. For one sample binomial interval estimation, the Blyth-Still-Casella interval was used, an exact confidence interval, that is considered more conservative or shorter in width than the commonly used Clopper-Pearson confidence intervals. ${ }^{7}$ Pair-wise comparisons were [performed using Tukey's HSD test. Multivariable logistic regression was used to adjust results for uterine rupture by potential confounders. These confounders included gestational age at delivery which was determined by the best obstetrical measurement assigned by the local caregivers, induction of labor defined as the administration of a drug such as oxytocin to initiate labor and maternal age at delivery.. Odds ratios (OR) and 95\% confidence intervals (CI) for outcomes were determined. Nominal two-sided probability values are reported with statistical significance defined as $\mathrm{P}<0.05$ without adjustments for multiple comparisons. Statistical analyses were performed using SAS (SAS Institute Inc, Cary, NC) and StatXact (Cytel Software, Cambridge, MA).

\section{RESULTS}

Out of 47,112 women in the Cesarean Registry, we identified 176 women who had a prior myomectomy, 13,273 with one prior LTC and 455 who had a prior classical cesarean that met the inclusion criteria for our study $(\mathrm{N}=13,904)$. Details regarding the excluded patients are shown in Figure 1. Of the final cohort, all patients in the P-MMX group delivered by cesarean, whereas 27 (5.9\%) in the PC and 5873 (44.2\%) in the LTC group delivered vaginally. Demographic and labor characteristics of our cohort differed between groups (Table 1). Women with P-MMX or PC cesareans were significantly more likely to be African-American, while those with a prior LTC were more likely to be Caucasian $(\mathrm{p}<0.001)$. Women in the P-MMX group were older compared with the other groups (Tukey pair-wise comparison, all $\mathrm{p}<0.05$ ). Finally, women with a prior LTC had the highest mean gestational age at delivery and were the most likely to have labored (all p<0.001).

The main outcome results are presented in Table 2. The frequency of uterine rupture risk in the P-MMX group $(0 \%, 95 \%$ CI $0-1.98 \%)$ was not significantly different from the risk in the LTC group $(0.41 \%, 95 \%$ CI $0.31-0.53 \%, \mathrm{p}>0.99)$. The frequency of rupture in the PC cesarean group $(0.88 \%, 95 \%$ CI $0.30-2.19 \%)$ also was not significantly different when compared with the LTC group, $(\mathrm{p}=0.13)$. However, after controlling for factors (maternal age, gestational age at delivery, and induction) that may influence the rate of rupture, we found that only a prior classical incision carried a higher risk of uterine rupture (OR 3.23, 95\% CI 1.11-9.39) compared with the LTC group (Table 3). Additionally, although the PC group was delivered earlier than those with P-MMX (35.8 v. 37.3 weeks), the frequency of uterine rupture in the 2 groups $(0.88$ vs. $0 \%)$ was not significantly different, $\mathrm{p}=0.58$. Twenty-one percent of P-MMX, 36.7\% of PC and 66.5\% of LTC women had signs of labor (i.e. regular contractions); the respective uterine rupture frequencies with labor were $0 \%$, $1.8 \%$ and $0.6 \%(\mathrm{p}=0.14)$. Also $1.1 \% \mathrm{P}-\mathrm{MMX}, 2.6 \% \mathrm{PC}$ and $17.8 \% \mathrm{LTC}$ had labor induction with respective rupture frequencies of $0 \%, 0 \%$ and $1.0 \%(\mathrm{p}>0.99)$. 
Results for placenta previa and placenta accreta by study groups are also presented in Table 2 . The frequency of placenta previa in the LTC group $(0.78 \%)$ was not different from the risk in the P-MMX group $(0.57 \%, \mathrm{p}>0.99)$ but it was significantly lower than the risk in the PC group $(1.98 \%, \mathrm{p}=0.01)$. However, after adjusting for potential confounders this association between PC cesarean and placenta previa disappeared. Additionally, the risks of previa in P-MMX and PC were not significantly different $(\mathrm{p}=0.30)$. The unadjusted overall risks of placenta accreta for the groups mirrored the relationship observed for placenta previa, including no statistical difference in the risks of P-MMX and PC $(\mathrm{p}=0.58)$. Also placenta accreta with previa occurred in $0 \%$ of P-MMX compared with $0.11 \%$ in the LTC group ( $p>.99)$. Accreta with previa was not different with PC (0.22\%; compared with LTC (0.11), $\mathrm{p}=0.40$. However, among those with previa, the frequencies of accreta were $0 \%$, $11.1 \%$ and $13.6 \%$ for the P-MMX, PC and LTC groups respectively although these were not statistically different from each other (all p>.99).

\section{DISCUSSION}

We found that women in the P-MMX group had risks of uterine rupture and accreta comparable with risks in women with a prior LTC or a PC. However, in the PC group a higher adjusted risk of uterine rupture was observed compared with the LTC group. Consistent with prior studies, ${ }^{8,9}$ women with LTC and a previa had the highest risk of placenta accreta, although we did not demonstrate a statistical difference vis-à-vis the other groups. The absolute risks of placenta accreta overall were low $(<1 \%)$ in all 3 groups.

There are a number of strengths to our study. As mentioned previously, this is one of the few studies to assess risks of uterine rupture and accreta in a relatively high number of women with a prior uterine surgery outside of the lower segment. The MFMU Cesarean Registry provides a comprehensive database of women with a prior cesarean uterine incision who are undergoing a subsequent pregnancy for comparison. However, we acknowledge several study limitations. We did not have access to all operative reports, particularly for the prior myomectomies, so we were not able to ascertain the location of the myomas (e.g. subserosal, intramural or submucosal), whether the endometrial cavities were entered, the extent of the prior surgeries (including number of myomas removed) or type of surgery (laparoscopy versus laparotomy). Only women with a prior MMX who underwent a cesarean delivery are included; any women with a prior myomectomy who delivered vaginally were not captured (although these are likely to be few and unlikely to materially impact our results). Also, only a negligible proportion of the included population had labor or induction and therefore our sample size is limited to address the risks of a trial of labor after prior myomectomy. While we cannot comment on whether uterine rupture would have occurred if the pregnancies had continued, despite a $20.5 \%$ rate of labor, there remained no ruptures in the P-MMX group.

Few, if any, studies have examined the risks of uterine rupture or accreta in women with a prior myomectomy or classical cesarean. Many clinicians believe that the risk of uterine rupture in women with a prior MMX is higher if the endometrial cavity was entered at the time of the myomectomy, and that the risk overall is similar to that seen with prior classical cesareans. However, there is a paucity of data to support this belief. Still, because of this assertion, women with a P-MMX are often delivered by cesarean to prevent the risk of uterine rupture as evidenced by the relatively comparably low prevalence of labor or labor induction in our series vis-à-vis the LTC group. Furthermore, women with a prior myomectomy are often delivered prior to 39 weeks (as demonstrated by our data), and often prior to 37 weeks, in order to prevent uterine rupture. A review of 412 women with a pregnancy after a 1,225 myomectomies at a teaching institution in Nigeria found that the incidence of uterine rupture was low $-0.24 \% .{ }^{4}$ Twenty-four of 67 women whose 
myomectomy dissection involved the endometrial cavity were allowed to labor. None experienced a uterine rupture, but the authors do not state how many went on to deliver vaginally. Similarly, there was a low rate of uterine rupture in our study. There are several case reports of second trimester uterine rupture for patients with a prior classical cesarean scar that have led to these concerns. ${ }^{10,11}$ Late preterm delivery of women with a P-MMX has become a common practice even though we observed no uterine rupture in the 176 women in our cohort.

In our cohort, the uterine rupture rate in women with a prior classical cesarean delivery was $0.88 \%$. This is in contrast to the rate of uterine rupture that has been quoted as $5-9 \%$ in women with a prior classical cesarean who labor. ${ }^{12}$ This is most likely related to efforts by practitioners to deliver these patients earlier and without labor or labor induction. However, this rate remained significantly high compared with women with a prior low transverse cesarean delivery.

Placenta accreta occurs when Nitabuch's layer is incompletely formed between the placenta and the myometrial layer. It is thought that a scarred uterus is less likely to have an intact Nitabuch's layer, increasing the risk for placenta accreta. This is corroborated by the finding that an increasing number of prior cesarean deliveries lead to an increase in the rate of accreta. ${ }^{6,9}$ Therefore we sought to estimate whether the risks of accreta were also increased in women with a prior myomectomy or prior classical cesarean. We found uniformly low rates of placenta accreta in these women although women in the PC but not the P-MMX may have a higher risk of accreta than the LTC group (albeit not statistically significant after multivariable adjustment). This supports the findings by Warshak et al, in their evaluation of the rates of accreta in 453 women with either placenta previa, a low-lying placenta, previous cesarean delivery or prior myomectomy. ${ }^{3}$ They found only one case of accreta in women with a prior myomectomy $(1 / 39,2.5 \%)$. This finding may be explained by the potentially larger area of disruption from a classical scar as opposed to the smaller myomectomy scar. Despite the lack of pathological confirmation of accreta, our findings are reassuring for women who have had either a prior myomectomy or prior classical delivery since rates of both uterine rupture and placenta accreta are low in our cohort.

In conclusion, the rates of both uterine rupture and placenta accreta are low in women with a prior myomectomy in our cohort. The low risks likely reflect practice patterns that include measures to prevent uterine rupture and other complications. However, considering there is little objective supporting evidence for an optimal delivery GA and considering the morbidity associated with late per term birth, we would advocate against delivery prior to 37 completed weeks. Women considering pregnancy after a prior myomectomy can be counseled with these data.

\section{Acknowledgments}

The project described was supported by grants from the Eunice Kennedy Shriver National Institute of Child Health and Human Development (NICHD) of the National Institutes of Health (NIH) [HD21410, HD21414, HD27860, HD27861, HD27869, HD27905, HD27915, HD27917, HD34116, HD34122, HD34136, HD34208, HD34210, HD40500, HD40485, HD40544, HD40545, HD40560, HD40512, and HD36801] and its contents are solely the responsibility of the authors and do not necessarily represent the official view of NICHD or the NIH.

The authors thank Francee Johnson, RN, BSN, for protocol development and coordination between clinical research centers; Elizabeth Thom, $\mathrm{PhD}$, for protocol and data management and statistical analysis; and Ronald J. Wapner, MD, and John C. Hauth, MD, for protocol development and oversight. 


\section{Appendix}

In addition to the authors, other members of the Eunice Kennedy Shriver National Institute of Child Health and Human Development Maternal-Fetal Medicine Units Network are as follows:

Columbia University — F. Malone, M. D’Alton, V. Pemberton, V. Carmona, H. Husami

University of Texas Southwestern Medical Center - L. Leveno, S. Bloom, J. Gold, D. Bradford

University of Utah - M. Belfort (Utah Valley Regional Medical Center), F. Porter (Intermountain Healthcare), B. Oshiro (McKay-Dee Hospital Center), K. Anderson (University of Utah Health Sciences Center), A. Guzman (McKay-Dee Hospital Center)

University of Pittsburgh - M. Cotroneo, D. Fischer, M. Luce

Wake Forest University Health Sciences - M. Harper, M. Swain, C. Moorefield, K. Lanier, L. Steele

The Ohio State University — J. Iams, F. Johnson, S. Meadows, H. Walker

Thomas Jefferson University — A. Sciscione, M. DiVito, M. Talucci, M. Pollock

Wayne State University - M. Dombrowski, G. Norman, A. Millinder, C. Sudz, B. Steffy

University of Cincinnati - M. Miodovnik, T. Siddiqi, H. How, N. Elder

Brown University - H. Silver, J. Tillinghast, D. Catlow, D. Allard

University of Alabama at Birmingham - J. Hauth, A. Northen, S. Tate

Northwestern University — M. Socol, D. Gradishar, G. Mallett

University of Miami - G. Burkett, J. Gilles, J. Potter, F. Doyle, S. Chandler

University of Tennessee - W. Mabie, R. Ramsey

University of Texas at San Antonio - O. Langer, D. Conway, S. Barker, M. Rodriguez

University of North Carolina - K. Moise, K. Dorman, S. Brody, J. Mitchell

The University of Texas Health Science Center at Houston — L. Gilstrap, M. Day, M. Kerr, E. Gildersleeve

University of Chicago - A. Moawad, J. Hibbard, P. Jones, M. Ramos-Brinson, M. Moran, D. Scott

Case Western Reserve University - P. Catalano, C. Milluzzi, B. Slivers, C. Santori

The George Washington University Biostatistics Center - E. Thom, H. JuliussenStevenson, M. Fischer, L. Leuchtenburg

Eunice Kennedy Shriver National Institute of Child Health and Human Development - D. McNellis, K. Howell, S. Tolivaisa 
MFMU Steering Committee Chair (Vanderbilt University Medical Center) - S. Gabbe

\section{REFERENCES}

1. Spong CY, Mercer BM, D’Alton M, Kilpatrick S, Blackwell S, Saade G. Timing of indicated latepreterm and early-term birth. Obstet Gynecol. 2011; 118:323-33. [PubMed: 21775849]

2. Landon MB, Lynch CD. Optimal timing and mode of delivery after cesarean with previous classical incision or myomectomy: a review of the data. Semin Perinatol. 2011; 35:257-61. [PubMed: 21962624]

3. Warshak CR, Eskander R, Hull AD, et al. Accuracy of ultrasonography and magnetic resonance imaging in the diagnosis of placenta accreta. Obstet Gynecol. 2006; 108:573-81. [PubMed: 16946217]

4. Obed JYOA. Rupture of the Uterus in Patients with Previous Myomectomy and Primary Caesarean Sectoion Scars: a Comparison. Journal of Obstetrics and Gynecology. 1996; 16:16-21.

5. Campo S, Campo V, Gambadauro P. Reproductive outcome before and after laparoscopic or abdominal myomectomy for subserous or intramural myomas. Eur J Obstet Gynecol Reprod Biol. 2003; 110:215-9. [PubMed: 12969587]

6. Landon MB, Hauth JC, Leveno KJ, et al. Maternal and perinatal outcomes associated with a trial of labor after prior cesarean delivery. N Engl J Med. 2004; 351:2581-9. [PubMed: 15598960]

7. Casela G. Refining binomial confidence intervals. Can J Stat. 1986; 14:113-29.

8. Grobman WA, Gersnoviez R, Landon MB, et al. Pregnancy outcomes for women with placenta previa in relation to the number of prior cesarean deliveries. Obstet Gynecol. 2007; 110:1249-55. [PubMed: 18055717]

9. Silver RM, Landon MB, Rouse DJ, et al. Maternal morbidity associated with multiple repeat cesarean deliveries. Obstet Gynecol. 2006; 107:1226-32. [PubMed: 16738145]

10. Endres LK, Barnhart K. Spontaneous second trimester uterine rupture after classical cesarean. Obstet Gynecol. 2000; 96:806-8. [PubMed: 11094213]

11. Levrant SG, Wingate M. Midtrimester uterine rupture. A case report. J Reprod Med. 1996; 41:186-90. [PubMed: 8778419]

12. Cunningham, FGHJ.; Leveno, KJ.; Gilstrap, LC., III; Bloom, SL.; Wenstrom, KD., editors. Williams Obstetrics. 22nd edition. McGraw-Hill; New York: 2005. 


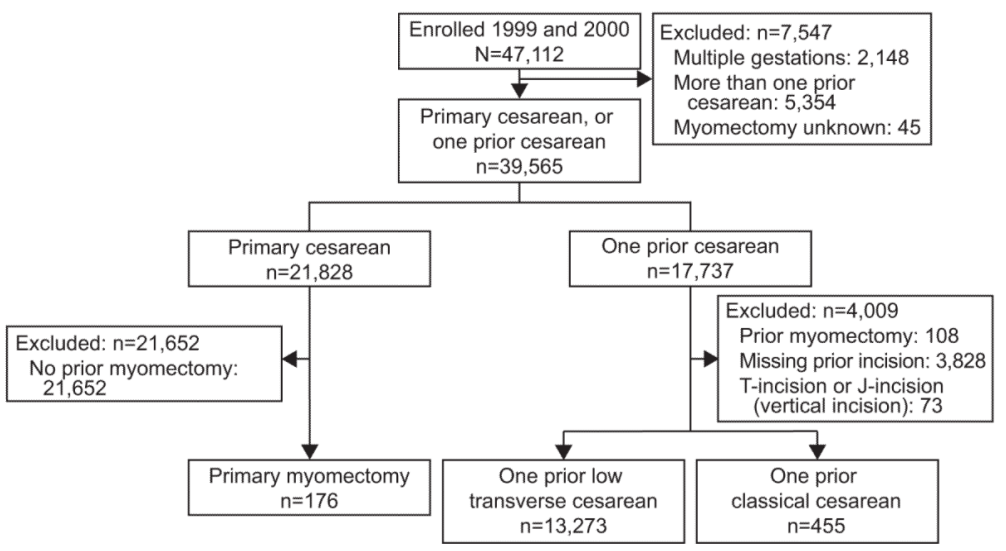

Figure 1.

Flow of patients included in this analysis. 
Table 1

Characteristics of the Study Population by the Type of Prior Uterine Scar

\begin{tabular}{|c|c|c|c|c|}
\hline & $\begin{array}{c}\text { Prior Myomectomy } \\
n=176\end{array}$ & $\begin{array}{c}\text { Prior Classical } \\
n=455\end{array}$ & $\begin{array}{l}\text { Prior Low Transverse } \\
\text { Cesarean } \\
\mathbf{n = 1 3 2 7 3}\end{array}$ & $P$ \\
\hline \multicolumn{5}{|l|}{ Race } \\
\hline African-American & 75 (42.6) & $223(49.0)$ & $4530(34.1)$ & $<0.001$ \\
\hline Caucasian & $52(29.5)$ & $132(29.0)$ & $5732(43.2)$ & \\
\hline Hispanic & $23(13.1)$ & $83(18.2)$ & 2373 (17.9) & \\
\hline Asian & $5(2.8)$ & $3(0.7)$ & $226(1.70)$ & \\
\hline Native American & $1(0.6)$ & $0(0)$ & $32(0.2)$ & \\
\hline Other & $20(11.4)$ & $14(3.1)$ & $380(2.90)$ & \\
\hline $\begin{array}{l}\text { Age } \\
\text { (years) }\end{array}$ & $\begin{array}{c}35.2 \pm 5.0 \\
35.0(32.5,39.0)\end{array}$ & $\begin{array}{c}28.8 \pm 6.1 \\
28.0(24.0,33.0)\end{array}$ & $\begin{array}{c}28.6 \pm 5.8 \\
29.0(24.0,33.0)\end{array}$ & $<0.001$ \\
\hline $\begin{array}{l}\text { Gestational age at } \\
\text { delivery (weeks) }\end{array}$ & $\begin{array}{c}37.3 \pm 2.9 \\
38.3(36.9,38.9)\end{array}$ & $\begin{array}{c}35.8 \pm 4.1 \\
37.1(34.1,38.4)\end{array}$ & $\begin{array}{c}38.6 \pm 2.7 \\
39.1(38.0,40.0)\end{array}$ & $<0.001$ \\
\hline Labor occurred & $36(20.5)$ & $167(36.7)$ & $8823(66.5)$ & $<0.001$ \\
\hline Induced & $2(1.1)$ & $12(2.6)$ & $2359(17.8)$ & $<0.001$ \\
\hline
\end{tabular}

IQR, interquartile range.

Data are $\mathrm{n}(\%)$ or mean \pm standard deviation with median and interquartile range unless otherwise specified. 
$N$
$\frac{0}{0}$
$\stackrel{0}{10}$ 
Table 3

Maternal Outcomes for Prior Classical Compared With Lower Transverse Cesarean Delivery

\begin{tabular}{|l|c|c|}
\hline & Odds Ratio (95\% CI) & Adjusted Odds Ratio (95\% CI) \\
\hline Rupture & $2.17(0.78-6.02)$ & $3.23(1.11-9.39)^{*}$ \\
Previa & $2.58(1.30-5.13)$ & $1.26(0.62-2.59)^{\dagger}$ \\
Accreta (any) & $4.70(1.63-13.56)$ & $2.09(0.69-6.33)^{\dagger}$ \\
Accreta (with previa) & $2.09(0.27-15.90)$ & $0.82(0.10-6.49)^{\dagger}$ \\
\hline
\end{tabular}

CI, confidence interval.

*Adjusted for maternal age, gestational age at delivery, labor induction.

${ }^{\dagger}$ Adjusted for maternal age and gestational age at delivery. 